\title{
A FLORISTIC INVENTORY OF THE UNIVERSITY OF OKLAHOMA'S KESSLER ATMOSPHERIC AND ECOLOGICAL FIELD STATION, MCCLAIN COUNTY, OKLAHOMA
}

\author{
Amy K. Buthod \\ Oklahoma Biological Survey \\ University of Oklahoma \\ Norman, OK 73019 \\ amybuthod@ou.edu
}

\author{
Bruce W. Hoagland \\ Oklahoma Biological Survey \\ Department of Geography and \\ Environmental Sustainablity \\ University of Oklahoma \\ Norman, OK 73019
}

Keywords: vascular, tare, non-native, tallgrass, prairie

\begin{abstract}
This paper reports the results of a vascular plant inventory at the University of Oklahoma's Kessler Atmospheric and Ecological Field Station in McClain County in the state of Oklahoma. A total of 388 taxa in 80 families were collected. Two hundred and fifty-seven genera, 361 species, and 27 infraspecific taxa were identified. The largest families were the Poaceae with 66 taxa and the Asteraceae with 55 taxa. Fifty-seven taxa were planted or non-native to the U.S. $(14.7 \%$ of the flora). Four taxa tracked by the Oklahoma Natural Heritage Inventory were found.

\section{INTRODUCTION AND STUDY AREA}

The Kessler Atmospheric and Ecological Field Station (KAEFS) at the University of Oklahoma was established on properties donated by Dr. Edwin Kessler from 1988 to 2011 (http:// kaefs.ou.edu/). Located approximately $28 \mathrm{~km}$ southwest of the Norman campus, KAEFS promotes and facilitates collaborative research and learning. The station hosts both formal and informal courses, workshops, and numerous meteorological and biological experiments, including a long-term global warming experiment. The goal of this work was to compile a complete list of vascular plant taxa present at the site to assist current and future KAEFS researchers in species identification and documentation.

KAEFS occupies 146 ha in McClain County in central Oklahoma approximately $13 \mathrm{~km}$ from the town of Washington (Figure 1). Latitudinal extent ranges from

$34^{\circ} 58^{\prime} 15.99^{\prime} \mathrm{N}$ to $34^{\circ} 59^{\prime} 10.61^{\prime \prime} \mathrm{N}$ and longitudinal extent from $97^{\circ} 30^{\prime} 32.88^{\prime \prime} \mathrm{W}$ to $97^{\circ} 31^{\prime} 42.63^{\prime \prime} \mathrm{W}$. Physiographically, the site is located within the Western Red-bed Plains geomorphic province, which consists of gently rolling hills of red sandstone and shale of Permian age (Curtis et al. 2008; Johnson 2008). Two soil associations predominate at KAEFS: the Nash-LucentGrant (deep to shallow, gently sloping to moderately steep loams over sandstone uplands) and the Port-Pulaski-Keokuk (deep, nearly level loamy soils on floodplains; Moebius and Sparwasser 1979). KAEFS is located within the subtropical humid (Cf) climate zone (Trewartha 1968), with a mean annual temperature of $16^{\circ} \mathrm{C}$. Low temperatures (to $3^{\circ} \mathrm{C}$ ) occur in January, while the warmest temperatures occur in July (to $28^{\circ} \mathrm{C}$; Oklahoma Climatological Survey 2016). The month of May is typically the wettest, with an average precipitation of $13.5 \mathrm{~cm}$. Mean annual precipitation is
\end{abstract}


$96.7 \mathrm{~cm}$ (Oklahoma Climatological Survey 2016). Elevation ranges from $332 \mathrm{~m}$ to $343 \mathrm{~m}$. The dominant potential vegetation type is tallgrass prairie (Duck and Fletcher 1943).

The KAEFS property has a long history of farming and livestock grazing. The first homestead was established in 1904, and crops such as cotton, sorghum, and wheat were grown on the property up until the early 1970s. The property has an equally long history of cattle grazing, and a small herd still roams the grasslands today (http://kaefs.ou.edu/). A population of the Mediterranean basin native Carthamus lanatus L. was discovered at the site in the mid1970s and grew to approximately 3000 plants by 1989 . It was eradicated in the early 1990s with a strict regime of hand-pulling (Kessler unpublished).

\section{METHODS}

Vouchers of vascular plant taxa encountered at KAEFS were made throughout the growing seasons (March through October) of 2013, 2014, and 2015. Vouchers for U.S. non-native or planted taxa were only collected from naturalized populations. Specimens were processed according to standard procedures. In addition to these vouchers, collections from an earlier, unpublished study were also examined to see if additional taxa had been collected. Manuals used for identification included Diggs et al. (1999) and Tyrl et al. (2015). Identifications were verified by comparison with specimens from the Robert Bebb Herbarium at the University of Oklahoma (OKL). Duration, growth habit, vegetation type, and nativity were determined using the PLANTS database (USDA-NRSC 2016) and Taylor and Taylor (1991). Classification and nomenclature follow Angiosperm Phylogeny Group III (Stevens 2001 onward) and the Integrated Taxonomic Information System (ITIS 2016). All specimens were deposited at OKL.

\section{RESULTS AND DISCUSSION}

A total of 388 taxa in 80 families were collected (Appendix). Two hundred and fifty-seven genera, 361 species, and 27 infraspecific taxa were identified. Two hundred and fifty-two taxa were perennials; there were 133 annuals and three biennials. The largest families were the Poaceae with 66 taxa and the Asteraceae with 55 taxa. Two-hundred and fifty-one taxa were forbs, and 91 were graminoids. There were 26 trees, 12 shrubs, and eight vines. Four taxa tracked by the Oklahoma Natural Heritage Inventory were found (Table 1). Threehundred and seventy-nine of the 388 taxa were collected by the authors. Nine additional taxa were found during an earlier survey by former KAEFS researcher Becky Sherry.

Fifty-seven taxa were planted and naturalized or non-native to the U.S. (14.7\% of the flora). Fifty-six of these were non-native. This number is high when compared to surveys from other Oklahoma grassland-dominated sites (Table 2), but it is not surprising given the land use history at KAEFS. Taxodium distichum, which is native to southeastern Oklahoma, was also found but was planted by the former property owner and has since naturalized. The Poaceae had the greatest number of exotic taxa with 13. The Fabaceae followed with nine exotics. Carthamus lanatus, reported from the property as late as 1991, was not relocated (Kessler 1987; Hoagland et al. 2012; Kessler unpublished).

The predominant vegetation type encountered at KAEFS was the Schizachyrium scoparium-Sorghastrum nutans association, a herbaceous vegetation type found throughout Oklahoma on uplands with well-drained soils. Associated taxa included Amorpha canescens, Dichanthelium oligosanthes var. oligosanthes, Panicum virgatum, and Symphotrichum ericoides var. ericoides (Hoagland 2000). 
We encountered three community types dominated by woody plants at KAEFS. The Populus deltoides-Ulmus americana-Celtis laevigata forest association was found on the bottomlands surrounding the property's larger order streams. This vegetation type is found frequently throughout the state (excluding the panhandle) on moist or wet soils along riparian corridors. Associated taxa found in this association included Carya illinoinensis, Symphoricarpos orbiculatus, and Toxicodendron radicans (Hoagland 2000). Small stands of Quercus muehlenbergii were encountered in mesic situations. Associated taxa included Amphicarpaea bracteata, Desmodium glutinosum, Elephantopus carolinianus, and Phryma leptostachya. Upland woodlands of the Juniperus virginianaSchizachyrium scoparium association intergraded with the grasslands and the riparian zones of low order streams. This vegetation type is common throughout Oklahoma (excluding the panhandle) and is the product of fire suppression and land-use change. Associated taxa included Andropogon virginicus, Cirsium altissimum, Quercus marilandica, and Smilax bona-nox (Hoagland 2000).

Herbaceous wetland vegetation was restricted to ponds and creek channels. Plants found in this type included Coleataenia anceps, Juncus torreyi, Lycopus americanus, Teucrium canadense, and the invasive aquatic Myriophyllum spicatum. Disturbed areas included an area around a barn, the parking lots, and gravel roads. Plants in these areas included Mollugo verticillata, Mublenbergia paniculata, Polygonum ramosissimum, and Solanum rostratum.

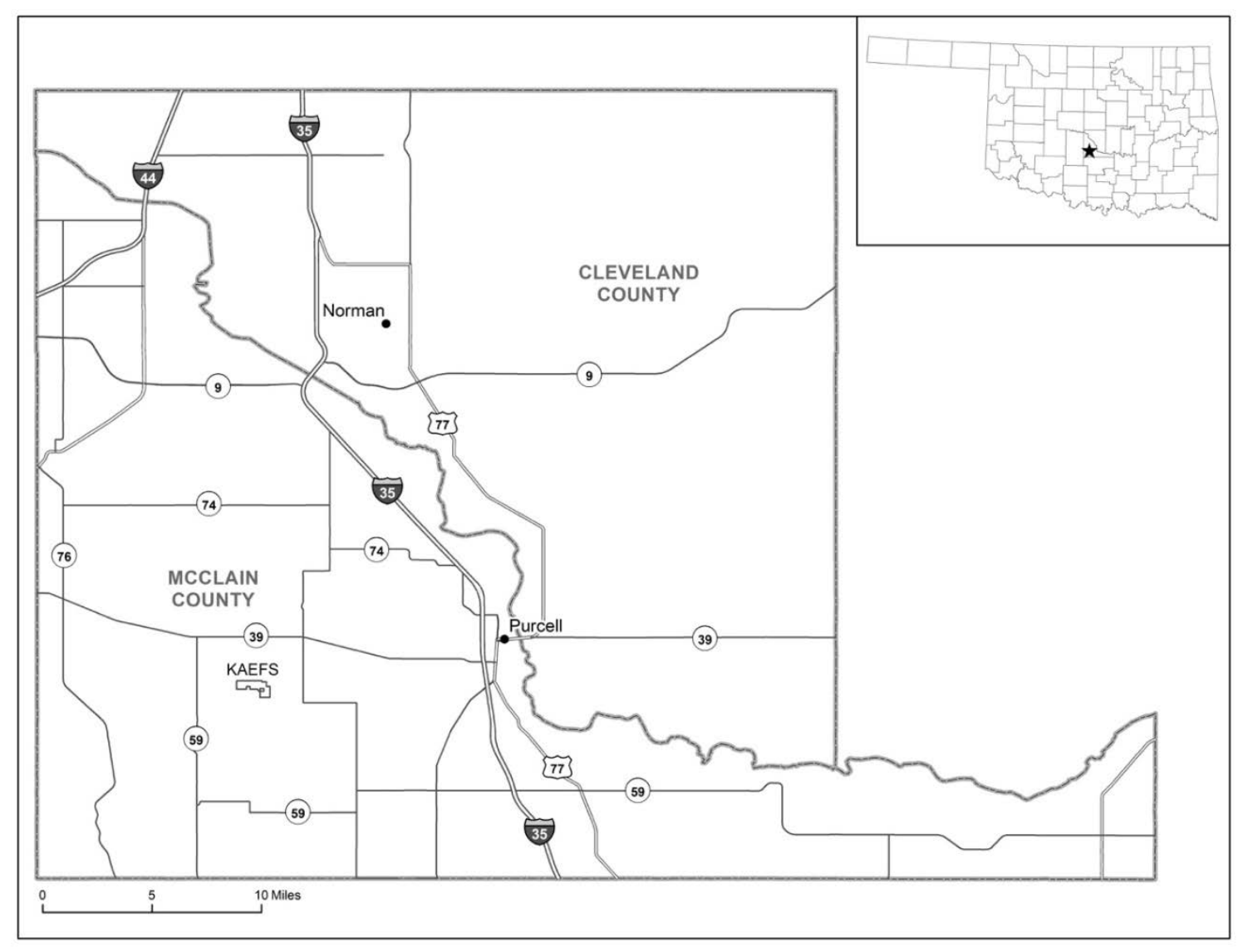

Figure 1 The Kessler Atmospheric and Ecological Field Station. Map by Todd Fagin, Oklahoma Biological Survey 
Table 1 Taxa located during this study that are tracked by the Oklahoma Natural Heritage Inventory (Groves 1995; NatureServe Explorer 2016; Oklahoma Natural Heritage Inventory 2016). Status ranks are on a 1-5 scale, with a 1 indicating the taxon is critically imperiled. $\mathrm{G}$ ranks are at the global level, and $\mathrm{S}$ ranks are at the subnational or state level. Infraspecific taxa are assigned a $\mathrm{T}$ rank.

\begin{tabular}{|l|l|l|}
\hline \multicolumn{1}{|c|}{ Family } & \multicolumn{1}{|c|}{ Taxon } & \multicolumn{1}{c|}{ Rank } \\
\hline Convolvulaceae & Ipomoea shumardiana (Torr.) Shinners & S3G2G3 \\
\hline Cyperaceae & Eleocharis geniculata (L.) Roem. \& Schult. & S2G5 \\
\hline Fabaceae & Desmodium nuttallii (Schindl.) B.G. Schub. & S1G5 \\
\hline Plantaginaceae & Plantago elongata Pursh ssp. elongata & S3T3G4T4 \\
\hline
\end{tabular}

Table 2 Comparison of exotic taxa from the KAEFS site with other Oklahoma grasslanddominated sites

\begin{tabular}{|l|l|c|c|c|}
\hline \multicolumn{1}{|c|}{ Study site } & \multicolumn{1}{|c|}{ Reference } & $\begin{array}{c}\text { Size of site } \\
\text { (ha) }\end{array}$ & $\begin{array}{c}\text { Number } \\
\text { of taxa } \\
\text { found }\end{array}$ & $\begin{array}{c}\text { Percentage of } \\
\text { non-native } \\
\text { taxa }\end{array}$ \\
\hline $\begin{array}{l}\text { Kessler Atmospheric and } \\
\text { Ecological Field Station, } \\
\text { McClain County }\end{array}$ & This paper & 146.0 & 388 & $14.7 \%$ \\
\hline $\begin{array}{l}\text { Pontotoc Ridge Nature } \\
\text { Preserve, Johnston and } \\
\text { Pontotoc Counties }\end{array}$ & $\begin{array}{l}\text { Buthod et al., } \\
\text { in preparation }\end{array}$ & 848.2 & 616 & $8.8 \%$ \\
\hline $\begin{array}{l}\text { Tulsa Botanic Garden, } \\
\text { Osage County }\end{array}$ & $\begin{array}{l}\text { Hoagland and } \\
\text { Buthod 2007 }\end{array}$ & 69.0 & 293 & $15.0 \%$ \\
\hline $\begin{array}{l}\text { Camp Kickapoo Boy } \\
\text { Scout Camp, Canadian } \\
\text { County }\end{array}$ & $\begin{array}{l}\text { Hoagland and } \\
\text { Buthod 2006 }\end{array}$ & 64.7 & 334 & $12.3 \%$ \\
\hline $\begin{array}{l}\text { Selman Living Laboratory, } \\
\text { Woodward County }\end{array}$ & $\begin{array}{l}\text { Buckallew and } \\
\text { Caddell 2003 }\end{array}$ & 129.5 & 229 & $9.0 \%$ \\
\hline
\end{tabular}




\section{ACKNOWLEDGEMENTS}

The authors wish to thank Todd Fagin, Daryn Hardwick, Jenna Messick, Abby Moore, Becky Sherry, Brenda Smith-Patten, and the Souza lab for assistance with specimen collection. We also thank Todd Fagin for his assistance with map preparation.

\section{LITERATURE CITED}

Buckallew, R.R. and G.M. Caddell. 2003. Vascular flora of the University of Central Oklahoma Selman Living Laboratory, Woodward County, Oklahoma. Proceedings of the Oklahoma Academy of Sciences 83:31-45.

Curtis, N.M., W.E. Ham, and K.S. Johnson. 2008. Geomorphic provinces of Oklahoma. In: Johnson, K.S. and K.V. Luza (eds.). Earth Sciences and Mineral Resources of Oklahoma. Norman (OK): Oklahoma Geological Survey.

Diggs, G.M., Jr., B.L. Lipscomb, and R.J. O'Kennon. 1999. Shinners and Mabler's Illustrated Flora of North Central Texas. Fort Worth (TX): Botanical Research Institute of Texas.

Duck, L.G. and J.B. Fletcher. 1943. A Game type map of Oklahoma. In: A Survey of the Game and Furbearing Animals of Oklahoma. Oklahoma City (OK): Oklahoma Department of Wildlife Conservation.

Groves, C.R., M.L. Klein, and T.F. Breden. 1995. Natural Heritage Programs: Public-private partnerships for biodiversity conservation. Wildlife Society Bulletin 23:784-790.

Hoagland, B.W. 2000. The vegetation of Oklahoma: A classification for landscape mapping and conservation planning. The Southwestern Naturalist 43:285-420.

Hoagland, B.W. and A.K. Buthod. 2007. The vascular flora of the Oklahoma Centennial Botanical Garden site, Osage
County, Oklahoma. Oklahoma Native

Plant Record 7:54-66.

Hoagland, B.W. and A. Buthod. 2006. Vascular flora of a red sandstone hills site, Canadian County, Oklahoma. Oklahoma Native Plant Record 6:53-68.

Hoagland, B.W., A.K. Buthod, I.H. Butler, P.H.C. Crawford, A.H. Udasi, W.J. Elisens, and R.J. Tyrl. 2004. Oklahoma Vascular Plants Database. http://www.oklahomaplantdatabase.org (22 August 2016).

Integrated Taxonomic Information System. 2016. www.ipni.org (6 June 2016).

Johnson, K.S. 2008. Generalized geologic map of Oklahoma. In: Johnson, K.S. and K.V. Luza (eds.). Earth Sciences and Mineral Resources of Oklahoma. Norman (OK): Oklahoma Geological Survey.

Kessler, E. 1987. Carthamus lanatus L. (Asteraceae: Cynareae) - a potentially serious plant pest in Oklahoma. Proceedings of the Oklahoma Academy of Science 67:39-43.

Moebius, G.E. and W.A. Sparwasser. 1979. Soil survey of McClain County, Oklahoma. Washington (D.C.): United States Department of Agriculture.

NatureServe. 2016. NatureServe Explorer. http://www.natureserve.org/explorer (1 August 2016).

Oklahoma Climatological Survey. 2016. The climate of McClain County. http://www.ocs.ou.edu (1 August 2016).

Oklahoma Natural Heritage Inventory. 2016. Vascular Plant Tracking List. http://www.biosurvey.ou.edu/downloa d/publications/TRACKING LIST JU L16.pdf (16 July 2016).

Stevens, P.F. (2001 onwards). Angiosperm Phylogeny Website. Version 12, July 2012. www.mobot.org/MOBOT/research/A Pweb (6 June 2016).

Taylor R.J. and C.E. Taylor. 1991. An Annotated List of the Ferns, Fern Allies, 
Gymnosperms, and Flowering Plants of Oklahoma. Durant (OK): Self-published. Trewartha, G.T. 1968. An Introduction to Climate. New York (NY): McGraw-Hill. Tyrl, R.J., S.C. Barber, P. Buck, W.J. Elisens, J.R. Estes, P. Folley, L.K. Magrath, C.L. Murray, A.K. Ryburn, B.A. Smith,
C.E.S. Taylor, R.A. Thomspon, J.B. Walker, and L.E. Watson. 2015. Flora of Oklahoma: Keys and Descriptions.

Oklahoma City (OK): Flora Oklahoma Inc.

USDA, NRCS. 2016. The PLANTS

Database. plants.usda.gov/plants (6 June 2016). 


\section{APPENDIX}

\section{List of Plant Taxa from the University of Oklahoma's Kessler Atmospheric and Ecological Field Station, McClain County, Oklahoma}

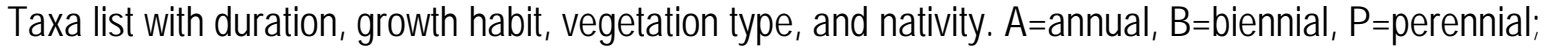
F=forb, G=graminoid, S=shrub, T=tree, V=woody vine; DA=disturbed area, GL=Schizachyrium scopariumSorghastrum nutans grassland, HWV=herbaceous wetland vegetation, MF=Quercus muehlenbergii mesic forest, SBL=Populus delotides-UImus americana-Celtis laevigata stream bottomland, UWL=Juniperus virginiana-Schizachyrium scoparium upland woodland. An asterisk $\left(^{*}\right)$ indicates a taxon that is non-native to the United States. A tilde ( ) indicates a taxon that is planted but native to Oklahoma. A dagger ( $\dagger$ ) indicates a tracked taxon. Duration and nativity were determined using the PLANTS Database (USDANRSC 2016); if the information from PLANTS was ambiguous, Taylor and Taylor (1991) was consulted. Common names were taken from PLANTS (USDA-NRSC 2016), and vegetation classifications were based on Hoagland (2000). All specimens were collected by the authors with the exception of those marked with the pound sign (\#), which were collected by Becky Sherry. Specimens were assigned collection numbers with the prefix KES.

\section{Acanthaceae}

Ruellia humilis Nutt. (denseflowered waterwillow); P; F; GL; KES-327

\section{Adoxaceae}

Viburnum rufidulum Raf. (rusty blackhaw); P; S; UWL; KES-077

\section{Amaranthaceae}

${ }^{*}$ Chenopodium album L. (lambsquarters); A; F; DA; KES-428

Chenopodium pratericola Rydb. (desert goosefoot); A; F; DA; KES-066

Iresine rhizomatosa Standl. (Juda's bush); P; F; SBL; KES-054

\section{Amaryllidaceae}

Allium canadense L. var. fraseri Ownbey (Fraser meadow garlic); P; F; GL; KES-152

Nothoscordum bivalve (L.) Britton (crow-poison); P; F; GL; KES-153

\section{Anacardiaceae}

Rhus copallinum L. (winged sumac); P; S; GL; KES-239

Rhus glabra L. (smooth sumac); P; S; GL; KES-052

Toxicodendron radicans (L.) Kuntze (eastern poison ivy); P; V; SBL; KES-051

\section{Apiaceae}

Ammoselinum butleri (Engelm. ex S. Watson) J.M. Coult. \& Rose (Butler's sandparsley); A; F; GL; KES-050 \#Ammoselinum popei Torr. \& A. Gray (plains sandparsley); A; F; GL; KES-407

Chaerophyllum tainturieri Hook. var. tainturieri (hairyfruit wild chervil); A; F; SBL; KES-087

Daucus pusillus Michx. (American wild carrot); A; F; GL; KES-047

Sanicula canadensis L. (snakeroot); B; F; MF; KES-048

Spermolepis inermis (Nutt. ex DC.) Mathias \& Constance (Red River scaleseed); A; F; GL; KES-049

*Torilis arvensis (Huds.) Link (hedge parsley); A; F; DA; KES-227 


\section{Apocynaceae}

Apocynum cannabinum L. (Indian hemp); P; F; GL; KES-046

Asclepias amplexicaulis Sm. (clasping milkweed); P; F; GL; KES-387

Asclepias stenophylla A. Gray (narrowleaf milkweed); P; F; GL; KES-041

Asclepias tuberosa L. (butterfly milkweed); P; F; GL; KES-040

Asclepias viridiflora Raf. (green comet milkweed); P; F; GL; KES-042

Asclepias viridis Walter (green milkweed); P; F; GL; KES-043

*Vinca major L. (greater periwinkle); P; F; SBL; KES-045

\section{Araliaceae}

*Hedera helix L. (English ivy); P; V; SBL; KES-044

\section{Asparagaceae}

Androstephium coeruleum (Scheele) Greene (blue funnel lily); P; F; GL; KES-154

Yucca glauca Nutt. (common yucca); P; S; GL; KES-393

\section{Aspleniaceae}

Asplenium platyneuron (L.) Britton, Sterns \& Poggenb. (ebony spleenwort); P; F; UWL; KES-379

\section{Asteraceae}

Achillea millefolium L. (yarrow); P; F; GL; KES-030

Ambrosia psilostachya DC. (western ragweed); P; F; GL; KES-004

Ambrosia trifida L. (giant ragweed); A; F; GL; KES-006

Amphiachyris dracunculoides (DC.) Nutt. (broomweed); A; F; GL; KES-014

\#Antennaria parlinii Fernald ssp. fallax (Greene) Bayer \& Stebbins (Parlin's pussytoes); P; F; MF; KES-408

Artemisia ludoviciana Nutt. ssp. mexicana (Willd. ex Spreng.) D.D. Keck (white sagebrush); P; F; GL;

KES-347

Berlandiera x betonicifolia (Hook.) Small (Texas greeneyes); P; F; GL; KES-008

Bidens bipinnata L. (Spanish needles); A; F; SBL; KES-012

Bradburia pilosa (Nutt.) Semple (golden aster); A; F; GL; KES-031

Cirsium altissimum (L.) Hill (tall thistle); B; F; UWL; KES-017

Cirsium undulatum (Nutt.) Spreng. (wavyleaf thistle); P; F; GL; KES-013

Conoclinium coelestinum (L.) DC. (mist flower); P; F; SBL; KES-019

Conyza canadensis (L.) Cronquist (horseweed); A; F; GL; KES-370

Conyza ramosissima Cronquist (dwarf horseweed); A; F; DA; KES-429

Diaperia verna (Raf.) Morefield (spring pygmycudweed); A; F; GL; KES-035

Echinacea angustifolia DC. (blacksamson); P; F; GL; KES-021

Eclipta prostrata (L.) L. (false daisy); A; F; HWV; KES-374

Elephantopus carolinianus Raeusch. (elephant's foot); P; F; MF; KES-376

Erechtites hieraciifolius (L.) Raf. ex DC. (American burnweed); A; F; GL; KES-423

Erigeron philadelphicus L.; (Philadelphia fleabane); P; F; SBL; KES-039

Erigeron strigosus Muhl. ex Willd. (prairie fleabane); A; F; GL; KES-003

Gaillardia aestivalis (Walter) H. Rock (summer gaillardia); P; F; GL; KES-027

Gamochaeta purpurea (L.) Cabrera (purple cudweed); P; F; GL; KES-313

Grindelia ciliata (Nutt.) Spreng. (wax goldenweed); A; F; GL; KES-011

Helianthus annuus L. (common sunflower); A; F; GL; KES-005

Helianthus maximiliani Schrad. (Maximilian sunflower); P; F; GL; KES-346 
Helianthus mollis Lam. (ashy sunflower); P; F; GL; KES-015 Heterotheca subaxillaris (Lam.) Britt. \& Rusby (camphorweed); A; F; GL; KES-344

Hieracium longipilum Torr. (longbeard hawkweed); P; F; GL; KES-001

Krigia caespitosa (Raf.) K.L. Chambers (common dwarf dandelion); A; F; GL; KES-395

Lactuca canadensis L. (Canada lettuce); A; F; SBL; KES-368

*Lactuca serriola L. (prickly lettuce); A; F; DA; KES-022

Liatris pycnostachya Michx. (button snakeroot); P; F; GL; KES-363

Liatris squarrosa (L.) Michx. (gayfeather); P; F; GL; KES-010

Pseudognaphalium obtusifolium (L.) Hilliard \& B.L. Burtt (rabbit-tobacco); A; F; GL; KES-009

Pyrrhopappus carolinianus (Walter) DC. (Carolina desert-chicory); A; F; GL; KES-294

Pyrrhopappus grandiflorus (Nutt.) Nutt. (false dandelion); P; F; GL; KES-026

Ratibida columnifera (Nutt.) Woot. \& Standl. (yellow coneflower); P; F; GL; KES-033

Rudbeckia hirta L. (black-eyed Susan); P; F; GL; KES-028

Silphium asteriscus L. (starry rosinweed); P; F; GL; KES-430

Solidago missouriensis Nutt. (Missouri goldenrod); P; F; GL; KES-034

Solidago radula Nutt. (rough goldenrod); P; F; GL; KES-357

Solidago rigida L. (stiff prairie goldenrod); P; F; GL; KES-366

*Sonchus asper (L.) Hill (spiny sowthistle); A; F; DA; KES-303

Symphyotrichum drummondii (Lindl.) G.L. Nesom var. texanum (E.S. Burgess) G.L. Nesom (blue wood

aster); P; F; UWL; KES-356

Symphyotrichum ericoides (L.) G.L. Nesom var. ericoides (heath aster); P; F; GL; KES-353

Symphyotrichum patens (Aiton) G.L. Nesom var. patens (spreading aster); P; F; GL; KES-425

Symphyotrichum subulatum (Michx.) G.L. Nesom (salt marsh aster); P; F; HWV; KES-018

*Taraxacum erythrospermum Andrz. ex Besser (rock dandelion); P; F; DA; KES-394

Tetraneuris linearifolia (Hook.) Greene (fineleaf fournerved daisy); A; F; GL; KES-037

\#Thelesperma filifolium (Hook.) A. Gray (greenthread); P; F; GL; KES-411

${ }^{*}$ Tragopogon dubius Scop. (yellow salsify); A; F; GL; KES-023

Verbesina virginica L. (Virginia crownbeard); P; F; SBL; KES-020

Vernonia baldwinii Torr. (western ironweed); P; F; GL; KES-007

Xanthium strumarium L. (cocklebur); A; F; HWV; KES-371

\section{Boraginaceae}

Lithospermum incisum Lehm. (narrowleaf puccoon); P; F; GL; KES-091

\section{Brassicaceae}

${ }^{*}$ Camelina microcarpa DC. (littlepod false flax); A; F; GL; KES-079

*Capsella bursa-pastoris (L.) Medik. (shepherd's purse); A; F; GL; KES-083

Cardamine pensylvanica Muhl. ex Willd. (Pennsylvania bittercress); A; F; SBL; KES-088

Descurainia pinnata (Walter) Britton (western tansymustard); A; F; GL; KES-089

Draba brachycarpa Nutt. ex Torr. \& A. Gray (shortpod draba); A; F; GL; KES-080

Draba cuneifolia Nutt. ex Torr. \& A. Gray (wedgeleaf draba); A; F; GL; KES-081

Lepidium austrinum Small (southern pepperweed); A; F; DA; KES-084

Lepidium oblongum Small (veiny pepperweed); A; F; GL; KES-082

Lepidium virginicum L. (Virginia peppergrass); A; F; GL; KES-078

Physaria ovalifolia (Rydb.) O'Kane \& Al-Shehbaz ssp. ovalifolia (roundleaf bladderpod); P; F; GL; KES-086

Rorippa palustris (L.) Besser ssp. palustris (bog yellowcress); A; F; HWV; KES-297 


\section{Cactaceae}

Escobaria missouriensis (Sweet) D.R. Hunt (Missouri foxtail cactus); P; F; GL; KES-250

Opuntia humifusa (Raf.) Raf. (devil's tongue); P; S; GL; KES-339

\section{Campanulaceae}

Triodanis perfoliata (L.) Nieuwl. ssp. perfoliata (clasping Venus' looking-glass); A; F; GL; KES-388

\section{Cannabaceae}

Celtis laevigata Willd. (sugarberry); P; T; SBL; KES-245

\section{Caprifoliaceae}

*Lonicera japonica Thunb. (Japanese honeysuckle); P; V; SBL; KES-076

Symphoricarpos orbiculatus Moench (coralberry); P; S; SBL; KES-075

\section{Caryophyllaceae}

*Arenaria serpyllifolia L. (thyme-leaved sandwort); A; F; GL; KES-072

*Cerastium pumilum W. Curtis (European chickweed); A; F; GL; KES-073

*Dianthus armeria L. (Deptford pink); A; F; GL; KES-068

Minuartia michauxii (Fenzl) Farw. (rock sandwort); P; F; GL; KES-382

Paronychia jamesii Torr. \& A. Gray (James' nailwort); P; F; GL; KES-069

Sagina decumbens (Elliott) Torr. \& A. Gray (beach pearlwort); A; F; GL; KES-071

Silene antirrhina L. (sleepy catchfly); A; F; GL; KES-067

*Stellaria media (L.) Vill. (common chickweed); A; F; SBL; KES-074

\section{Commelinaceae}

Commelina erecta L. (whitemouth dayflower); P; F; SBL; KES-065

Tradescantia occidentalis (Britton) Smyth (prairie spiderwort); P; F; GL; KES-316

\section{Convolvulaceae}

Cuscuta cuspidata Engelm. (cusp dodder); P; F; GL; KES-055

Cuscuta pentagona Engelm. (fiveangled dodder); A; F; GL; KES-056

†Ipomoea shumardiana (Torr.) Shinners (narrowleaf morning-glory); P; F; GL; KES-064; S3G2G3

\section{Cornaceae}

Cornus drummondii C.A. Mey. (rough-leaf dogweed); P; T; SBL; KES-062

\section{Cucurbitaceae}

Cucurbita foetidissima Kunth (buffalo gourd); P; F; GL; KES-240

Melothria pendula L. (Guadeloupe cucumber); P; F; DA; KES-059

\section{Cupressaceae}

Juniperus virginiana L. (eastern red cedar); P; T; UWL; KES-057

Taxodium distichum (L.) Rich. (bald cypress); P; T; SBL; KES-431

\section{Cyperaceae}

Carex aureolensis Steud. (goldenfruit sedge); P; G; HWV; KES-139

Carex blanda Dewey (eastern woodland sedge); P; G; UWL; KES-358 
Carex brevior (Dewey) Mack. (shortbeak sedge); P; G; GL; KES-402

Carex cephalophora Muhl. ex Willd. (oval-leaf sedge); P; G; MF; KES-403

Carex gravida L.H. Bailey (heavy sedge); P; G; GL; KES-321

Cyperus acuminatus Torr. \& Hook. ex Torr. (taperleaf flatsedge); P; G; HWV; KES-319

Cyperus echinatus (L.) Alph. Wood (globe flatsedge); P; G; GL; KES-138

*\#Cyperus esculentus L. (chufa flatsedge); P; G; DA; KES-406

Cyperus lupulinus (Spreng.) Marcks (Great Plains flatsedge); P; G; GL; KES-134

Cyperus squarrosus L. (bearded flatsedge); A; G; HWV; KES-140

†Eleocharis geniculata (L.) Roem. \& Schult. (Canada spikesedge); A; G; HWV; KES-335

Eleocharis montevidensis Kunth (sand spikesedge); P; G; HWV; KES-320

Eleocharis obtusa (Willd.) Schult. (blunt spikesedge); A; G; HWV; KES-382

Eleocharis palustris (L.) Roem. \& Schult. (common spikerush); P; G; HWV; KES-137

Fimbristylis puberula (Michx.) Vahl var. puberula (hairy fimbry); P; G; GL; KES-336

Fuirena simplex Vahl var. aristulata (Torr.) Kral (umbrella sedge); P; G; HWV; KES-331

Lipocarpha drummondii (Nees) G.C. Tucker (Drummond's halfchaff sedge); A; G; HWV; KES-325

Schoenoplectus pungens (Vahl) Palla var. longispicatus (Britton) S.G. Sm. (common threesquare); P; G; HWV; KES-136

Scirpus atrovirens Willd. (darkgreen bulrush); P; G; HWV; KES-135

Scleria ciliata Nees (fringed nutrush); P; G; GL; KES-301

\section{Ebenaceae}

Diospyros virginiana L. (persimmon); P; T; SBL; KES-230

\section{Elaeagnaceae}

*Elaeagnus umbellata Thunb. (oleaster); P; S; SBL; KES-169

\section{Equisetaceae}

Equisetum hyemale L. ssp. affine (Engelm.) Calder \& Roy (scouring horsetail); P; F; SBL; KES-133

\section{Euphorbiaceae}

Acalypha ostryifolia Riddell (pineland threeseed mercury); A; F; SBL; KES-122

Acalypha virginica L. (Virginia threeseeded mercury); A; F; SBL; KES-131

Croton capitatus Michx. (wooly croton); A; F; GL; KES-123

Croton glandulosus L. (croton); A; F; GL; KES-121

Croton monanthogynus Michx. (one-seed croton); A; F; GL; KES-127

Euphorbia corollata L. (flowering spurge); P; F; GL; KES-124

Euphorbia dentata Michx. (toothed spurge); A; F; SBL; KES-120

Euphorbia maculata L. (spotted sandmat); A; F; GL; KES-129

Euphorbia marginata Pursh (snow on the mountain); A; F; GL; KES-125

Euphorbia nutans Lag. (eyebane); A; F; GL; KES-373

Euphorbia prostrata Aiton (prostrate sandmat); A; F; GL; KES-132

Stillingia sylvatica L. (queen's delight); P; F; GL; KES-126

Tragia betonicifolia Nutt. (betonyleaf noseburn); P; F; GL; KES-130

\section{Fabaceae}

Amorpha canescens Pursh (leadplant); P; F; GL; KES-098

Amorpha fruticosa L. (false indigo); P; S; HWV; KES-381 
Amphicarpaea bracteata (L.) Fernald (hog peanut); A; F; MF; KES-112

Astragalus lotiflorus Hook. (lotus milkvetch); P; F; GL; KES-115

Baptisia australis (L.) R. Br. (blue wild indigo); P; F; GL; KES-390

Baptisia leucophaea Nutt. (plains wild indigo); P; F; GL; KES-118

Cercis canadensis L. (redbud); P; T; UWL; KES-119

Chamaecrista fasciculata (Michx.) Greene (partridge pea); A; F; GL; KES-097

Dalea aurea Nutt. ex Fraser (golden prairie clover); P; F; GL; KES-104

Dalea candida Michx. ex Willd. (white prairie clover); P; F; GL; KES-101

Dalea enneandra Nutt. ex Fraser (nine-anther prairie clover); P; F; GL; KES-092

Dalea purpurea Vent. (purple prairie clover); P; F; GL; KES-107

Desmanthus illinoensis (Michx.) MacMill. ex B.L. Rob. \& Fernald (bundleflower); P; F; GL; KES-111

Desmodium glutinosum (Muhl. ex Willd.) Alph. Wood (large-flowered tickclover); P; F; MF; KES-249

†Desmodium nuttallii (Schindl.) B.G. Schub. (Nuttall's ticktrefoil); P; F; GL; KES-113; S1G5

Desmodium sessilifolium (Torr.) Torr. \& A. Gray (sessile tickclover); P; F; GL; KES-105

Indigofera miniata Ortega (coastal indigo); P; F; GL; KES-110

*Kummerowia stipulacea (Maxim.) Makino (Korean clover); A; F; GL; KES-305

*Lathyrus hirsutus L. (Caley pea); A; F; GL; KES-306

Lespedeza capitata Michx. (bush clover); P; F; GL; KES-378

*Lespedeza cuneata (Dum. Cours.) G. Don (sericea lespedeza); P; F; GL; KES-103

Lespedeza procumbens Michx. (trailing lespedeza); P; F; MF; KES-367

Lespedeza stuevei Nutt. (Stueve's lespedeza); P; F; UWL; KES-369

*Medicago minima (L.) L. ex Bartal. (small medic); A; F; GL; KES-117

*Melilotus albus Medik. (white sweetclover); A; F; GL; KES-432

*Melilotus officinalis (L.) Lam. (yellow sweetclover); A; F; GL; KES-096

Mimosa nuttallii (DC. ex Britton \& Rose) B.L. Turner (sensitive briar); P; F; GL; KES-094

Oxytropis lambertii Pursh (purple locoweed); P; F; GL; KES-386

Psoralidium tenuiflorum (Pursh) Rydb. (wild alfalfa); P; F; GL; KES-100

Robinia pseudoacacia L. (black locust); P; T; UWL; KES-114

*Securigera varia (L.) Lassen (crownvetch); P; F; GL; KES-109

Strophostyles helvola (L.) Elliott (wild bean); A; F; GL; KES-106

Strophostyles leiosperma (Torr. \& A. Gray) Piper (smoothseed wild bean); A; F; GL; KES-377

*Vicia sativa L. (common vetch); A; F; GL; KES-116

*Vicia villosa Roth (winter vetch); A; F; GL; KES-396

\section{Fagaceae}

Quercus macrocarpa Michx. (bur oak); P; T; MF; KES-235

Quercus marilandica Münchh. (blackjack oak); P; T; UWL; KES-231

Quercus muehlenbergii Engelm. (chinquapin oak); P; T; MF; KES-234

\section{Gentianaceae}

Eustoma exaltatum (L.) Salisb. ex G. Don ssp. russellianum (Hook.) Kartesz (showy prairie gentian); A; F; GL; KES-375

Sabatia campestris Nutt. (pink gentian); A; F; GL; KES-150

\section{Geraniaceae}

${ }^{\star}$ Erodium cicutarium (L.) L'Hér. ex Aiton (redstem stork's bill); A; F; DA; KES-149

Geranium texanum (Trel.) A. Heller (Texas geranium); A; F; GL; KES-148

Amy K. Buthod and Bruce W. Hoagland 
Volume 16, December 2016

\section{Haloragaceae}

*Myriophyllum spicatum L. (Eurasian water-milfoil); P; F; HWV; KES-341

\section{Heliotropiaceae}

Heliotropium tenellum (Nutt.) Torr. (pasture heliotrope); A; F; GL; KES-090

\section{Hydrocharitaceae}

Najas guadalupensis (Spreng.) Magnus (southern waternymph); A; F; HWV; KES-311

\section{Hypericaceae}

Hypericum drummondii (Grev. \& Hook.) Torr. \& A. Gray (nits-and-lice); A; F; GL; KES-380

\section{Iridaceae}

*Iris germanica L. (German iris); P; F; SBL; KES-439

Sisyrinchium angustifolium Mill. (blue-eyed grass); P; F; GL; KES-146

Juglandaceae

Carya illinoinensis (Wangenh.) K. Koch; (pecan); P; T; SBL; KES-323

\section{Juncaceae}

Juncus brachyphyllus Wiegand (tuftedstem rush); P; G; HWV; KES-145

Juncus dudleyi Wiegand (Dudley's rush); P; G; GL; KES-398

Juncus interior Wiegand (inland rush); P; G; GL; KES-397

Juncus marginatus Rostk. (grassleaf rush); P; G; HWV; KES-144

Juncus torreyi Coville (Torrey's rush); P; G; HWV; KES-143

\section{Krameriaceae}

Krameria lanceolata Torr. (trailing ratany); P; F; GL; KES-164

\section{Lamiaceae}

Hedeoma drummondii Benth. (false pennyroyal); P; F; GL; KES-161

Hedeoma hispida Pursh (rough pennyroyal); A; F; GL; KES-159

*Lamium amplexicaule L. (henbit deadnettle); A; F; GL; KES-162

*Lamium purpureum L. (purple deadnettle); A; F; SBL; KES-163

Lycopus americanus Muhl. ex W.P.C. Bartram (American bugleweed); P; F; HWV; KES-158

Monarda clinopodioides A. Gray (basil beebalm); A; F; GL; KES-160

Monarda fistulosa L. (wild bergamot); P; F; GL; KES-156

Salvia azurea Michx. ex Lam. (blue sage); P; F; GL; KE-157

Teucrium canadense L. (American germander); P; F; HWV; KES-155

\section{Linaceae}

Linum sulcatum Riddell (grooved flax); A; F; GL; KES-151

\section{Lythraceae}

Ammannia coccinea Rottb. (valley redstem); A; F; HWV; KES-364 


\section{Malvaceae}

Callirhoe involucrata (Torr. \& A. Gray) A. Gray (winecup); P; F; GL; KES-168

\section{Menispermaceae}

Cocculus carolinus (L.) DC. (Carolina snailseed); P; F; UWL; KES-233

\section{Molluginaceae}

Mollugo verticillata L. (green carpetweed); A; F; DA; KES-167

\section{Montiaceae}

Claytonia virginica L. (springbeauty); P; F; GL; KES-282

Phemeranthus parviflorus (Nutt.) Kiger (sunbright); P; F; GL; KES-317

\section{Moraceae}

Maclura pomifera (Raf.) C.K. Schneid. (bois d'arc); P; T; SBL; KES-248

*Morus alba L. (white mulberry); P; T; DA; KES-166

Morus rubra L. (red mulberry); P; T; SBL; KES-165

\section{Nyctaginaceae}

Mirabilis albida (Walter) Heimerl (white four o'clock); P; F; GL; KES-361

Mirabilis linearis (Pursh) Heimerl (narrowleaf four o'clock); P; F; GL; KES-383

\section{Oleaceae}

Fraxinus pennsylvanica Marsh. (green ash); P; T; SBL; KES-379

\section{Onagraceae}

Oenothera berlandieri (Spach) Steud. ssp. berlandieri (Spach) Steud. (Berlandier's sundrops); P; F; GL;

KES-218

Oenothera curtiflora W.L. Wagner \& Hoch (velvety gaura); A; F; GL; KES-217

Oenothera glaucifolia W.L. Wagner \& Hoch (false gaura); P; F; GL; KES-221

Oenothera laciniata Hill (cut-leaf evening primrose); P; F; GL; KES-220

Oenothera macrocarpa Nutt. (large-fruited evening primrose); P; F; GL; KES-219

Oenothera rhombipetala Nutt. ex Torr. \& A. Gray (fourpoint evening primrose); P; F; GL; KES-222

Oenothera sinuosa W.L. Wagner \& Hoch (wavyleaf gaura); P; F; GL; KES-223

Oenothera triangulata (Buckley) W.L. Wagner \& Hoch (prairie beeblossom); A; F; GL; KES-391

\section{Ophioglossaceae}

Ophioglossum engelmannii Prantl (limestone adder's tongue); P; F; GL; KES-216

\section{Orchidaceae}

Spiranthes cernua (L.) Rich. (nodding lady's tresses); P; F; GL; KES-385

\section{Orobanchaceae}

Agalinis heterophylla (Nutt.) Small (prairie false foxglove); A; F; GL; KES-381

Buchnera americana L. (American blue hearts); P; F; GL; KES-266

Castilleja indivisa Engelm. (Indian paintbrush); A; F; GL; KES-267 
Volume 16, December 2016

\section{Oxalidaceae}

Oxalis corniculata L. (yellow wood-sorrel); P; F; GL; KES-215

Oxalis violacea L. (violet wood-sorrel); P; F; GL; KES-384

\section{Penthoraceae}

Penthorum sedoides L. (ditch stonecrop); P; F; HWV; KES-061

\section{Phyrmaceae}

Phryma leptostachya L. (American lopseed); P; F; MF; KES-293

\section{Phytolaccaceae}

Phytolacca americana L. (pokeweed); P; F; DA; KES-334

\section{Plantaginaceae}

Leucospora multifida (Michx.) Nutt. (narrowleaf paleseed); A; F; HWV; KES-265

Nuttallanthus texanus (Scheele) D.A. Sutton (Texas toadflax); B; F; GL; KES-392

Penstemon cobaea Nutt. (large beardtongue); P; F; GL; KES-385

Plantago aristata Michx. (bottlebrush plantain); A; F; GL; KES-289

†Plantago elongata Pursh ssp. elongata (prairie plantain); A; F; GL; KES-290; S3T3G4T4

Plantago patagonica Jacq. (wooly plantain); A; F; GL; KES-309

Plantago rhodosperma Decne. (redseed plantain); A; F; GL; KES-291

Plantago rugelii Decne. (blackseed plantain); P; F; SBL; KES-433

*Veronica arvensis L. (common speedwell); A; F; GL; KES-268

*\#Veronica peregrina L. (purslane speedwell); A; F; GL; KES-410

\section{Platanaceae}

Platanus occidentalis L. (American sycamore); P; T; SBL; KES-389

\section{Poaceae}

*Aegilops cylindrica Host (jointed goatgrass); A; G; GL; KES-170

Agrostis hyemalis (Walter) Britton, Sterns \& Poggenb. (ticklegrass); P; G; GL; KES-214

Andropogon gerardii Vitman (big bluestem); P; G; GL; KES-213

Andropogon ternarius Michx. (splitbeard bluestem); P; G; GL; KES-351

Andropogon virginicus L. (broomsedge); P; G; GL; KES-424

Aristida longespica Poir. (slimspike threeawn); A; G; GL; KES-178

Aristida purpurea Nutt. var. Iongiseta (Steud.) Vasey (Fendler threeawn); P; G; GL; KES-211

Aristida purpurea Nutt var. purpurea (purple threeawn); P; G; GL; KES-175

*Bothriochloa ischaemum (L.) Keng (yellow bluestem); P; G; GL; KES-210

Bothriochloa laguroides (DC.) Herter var. torreyana (Steud.) M. Marchi \& Longhi-Wagner (silver beardgrass); P; G; GL; KES-209

Bouteloua curtipendula (Michx.) Torr. (sideoats grama); P; G; GL; KES-208

Bouteloua dactyloides (Nutt.) Columbus (buffalo grass); P; G; GL; KES-376

Bouteloua gracilis (Kunth) Lag. ex Griffiths (blue grama); P; G; GL; KES-207

Bouteloua hirsuta Lag. (hairy grama); P; G; GL; KES-206

Bouteloua rigidiseta (Steud.) Hitchc. (Texas grama); P; G; GL; KES-205

*Bromus arvensis L. (field brome); A; G; GL; KES-204

*Bromus catharticus Vahl (rescue grass); A; G; GL; KES-173 
Bromus pubescens Muhl. ex Willd. (Canada brome); P; G; UWL; KES-203

*Bromus tectorum L. (cheatgrass); A; G; GL; KES-174

Cenchrus spinifex Cav. (coastal sandbur); P; G; GL; KES-383

Chasmanthium latifolium (Michx.) H.O. Yates (inland sea oats); P; G; SBL; KES-202

Chloris verticillata Nutt. (windmill grass); P; G; GL; KES-201

Chloris virgata Sw. (feather fingergrass); A; G; GL; KES-434

Coleataenia anceps (Michx.) Soreng (beaked panicgrass); P; G; HWV; KES-187

${ }^{*}$ Cynodon dactylon (L.) Pers. (Bermuda grass); P; G; GL; KES-228

Dichanthelium acuminatum (Sw.) Gould \& C.A. Clark var. lindheimeri (Nash) Gould \& C.A. Clark

(Lindheimer panicgrass); P; G; GL; KES-296

Dichanthelium malacophyllum (Nash) Gould (softleaf rosette grass); P; G; UWL; KES-194

Dichanthelium oligosanthes (Schult.) Gould var. oligosanthes (Heller's rosette grass); P; G; GL; KES-193

Dichanthelium sphaerocarpon (Elliott) Gould (roundseed panicgrass); P; G; GL; KES-426

Digitaria ciliaris (Retz.) Pers. (southern crabgrass); A; G; GL; KES-435

Digitaria cognata (Schult.) Pilg. (fall witchgrass); P; G; GL; KES-198

Distichlis spicata (L.) Greene (saltgrass); P; G; GL; KES-378

Echinochloa muricata (P. Beauv.) Fernald (rough barnyard grass); A; G; HWV; KES-298

Elymus canadensis L. (Canada wildrye); P; G; GL; KES-197

Elymus virginicus L. (Virginia wild rye); P; G; UWL; KES-196

*Eragrostis cilianensis (Bellardi) Vignolo ex Janch. (stinkgrass); A; G; GL; KES-384

Eragrostis curtipedicellata Buckley (gummy lovegrass); P; G; GL; KES-192

*Eragrostis curvula (Schrad.) Nees (weeping lovegrass); P; G; GL; KES-329

Eragrostis hirsuta (Michx.) Nees (bigtop lovegrass); P; G; GL; KES-191

Eragrostis secundiflora J. Presl ssp. oxylepis (Torr.) S.D. Koch (red lovegrass); P; G; GL; KES-190

Eragrostis sessilispica Buckley (tumble lovegrass); P; G; GL; KES-200

\#Eragrostis trichodes (Nutt.) Alph. Wood (sand lovegrass); P; G; GL; KES-412

Hordeum pusillum Nutt. (little barley); A; G; GL; KES-377

Leersia virginica Willd. (whitegrass); P; G; HWV; KES-189

*Lolium perenne L. (perennial ryegrass); P; G; GL; KES-188

Mnesithea cylindrica (Michx.) de Koning \& Sosef (mousetail); P; G; GL; KES-199

\#Muhlenbergia capillaris (Lam.) Trin. (hairawn muhly); P; G; GL; KES-199

Muhlenbergia paniculata (Nutt.) Columbus (tumblegrass); P; G; DA; KES-330

Panicum capillare L. (witchgrass); A; G; GL; KES-186

Panicum philadelphicum Bernh. ex Trin. (Philadelphia witchgrass); A; G; GL; KES-345

Panicum virgatum L. (switchgrass); P; G; GL; KES-185

*Paspalum dilatatum Poir. (Dallis grass); P; G; GL; KES-184

Paspalum distichum Houtt. (knotgrass); P; G; GL; KES-183

Paspalum setaceum Michx. (thin paspalum); P; G; GL; KES-182

Phalaris caroliniana Walter (maygrass); A; G; GL; KES-386

${ }^{*}$ Poa annua L. (annual bluegrass); A; G; GL; KES-172

Schizachyrium scoparium (Michx.) Nash (little bluestem); P; G; GL; KES-181

Setaria parviflora (Poir.) Kerguélen (knotroot foxtail); P; G; GL; KES-326

*Setaria viridis (L.) P. Beauv. (green bristlegrass); A; G; GL; KES-315

Sorghastrum nutans (L.) Nash (Indian grass); P; G; GL; KES-372

*Sorghum halepense (L.) Pers. (Johnson grass); P; G; GL; KES-180

Sphenopholis obtusata (Michx.) Scribn. (prairie wedgescale); P; G; HWV; KES-176

Sporobolus cryptandrus (Torr.) A. Gray (sand dropseed); P; G; GL; KES-314

Amy K. Buthod and Bruce W. Hoagland 
Sporobolus pyramidatus (Lam.) Hitchc. (Madagascar dropseed); P; G; GL; KES-179

Tridens flavus (L.) Hitchc. (purpletop); P; G; GL; KES-177

Vulpia octoflora (Walter) Rydb. (poverty grass); A; G; GL; KES-171

\section{Polygalaceae}

Polygala alba Nutt. (white milkwort); P; F; GL; KES-288

Polygala incarnata L. (pink milkwort); A; F; GL; KES-287

Polygala verticillata L. (whorled milkwort); A; F; GL; KES-286

\section{Polygonaceae}

Eriogonum annuum Nutt. (annual buckwheat); A; F; GL; KES-355

Eriogonum longifolium Nutt. (longleaf buckwheat); P; F; GL; KES-284

*Fallopia convolvulus (L.) Á. Löve (black bindweed); A; F; GL; KES-312

Persicaria lapathifolia (L.) Gray (pale smartweed); A; F; HWV; KES-229

Persicaria punctata (Elliott) Small (dotted smartweed); A; F; HWV; KES-285

Polygonum ramosissimum Michx. (bushy knotweed); A; F; DA; KES-360

${ }^{*}$ Rumex crispus L. (curly dock); P; F; HWV; KES-283

\#Rumex hastatulus Baldwin (heartwing sorrel); P; F; GL; KES-409

\section{Potamogetonaceae}

Potamogeton nodosus Poir. (longleaf pondweed); P; F; HWV; KES-322

\section{Primulaceae}

Samolus valerandi L. (smallflower brookweed); P; F; HWV; KES-295

\section{Ranunculaceae}

Anemone berlandieri Pritz. (ten-petal windflower); P; F; GL; KES-281

Delphinium carolinianum Walter ssp. virescens (Nutt.) R.E. Brooks (Carolina larkspur); P; F; GL; KES-391

\section{Rosaceae}

Geum canadense Jacq. (white avens); P; F; MF; KES-304

*Potentilla recta L. (erect cinquefoil); P; F; GL; KES-280

Prunus angustifolia Marshall (Chickasaw plum); P; S; GL; KES-226

Prunus mexicana S. Watson (Mexican plum); P; T; GL; KES-241

*Pyrus calleryana Decne. (Callery pear); P; T; UWL; KES-278

Rosa foliolosa Nutt. ex Torr. \& A. Gray (prairie rose); P; F; GL; KES-224

${ }^{*}$ Rosa multiflora Thunb. (mulitflora rose); P; V; SBL; KES-277

Rubus pensilvanicus Poir. (Oklahoma blackberry); P; S; GL; KES-436

\section{Rubiaceae}

Cephalanthus occidentalis L. (buttonbush); P; S; HWV; KES-337

*Cruciata pedemontana (Bellardi) Ehrend. (piedmont bedstraw); A; F; GL; KES-275

Diodella teres (Walter) Small (poor-joe); A; F; GL; KES-272

Galium aparine L. (catchweed bedstraw); A; F; GL; KES-276

Galium virgatum Nutt. (southwest bedstraw); A; F; GL; KES-271

Houstonia pusilla Schoepf (prairie bluets); A; F; GL; KES-274

Stenaria nigricans (Lam.) Terrell var. nigricans (diamond flowers); P; F; GL; KES-273 


\section{Salicaceae}

Populus deltoides W. Bartram ex Marshall (cottonwood); P; T; SBL; KES-236

Salix exigua Nutt. (narrowleaf willow); P; T; HWV; KES-270

Salix nigra Marshall (black willow); P; T; HWV; KES-269

\section{Sapindaceae}

Acer negundo L. (boxelder); P; T; SBL; KES-247

Sapindus saponaria L. var. drummondii (Hook. \& Arn.) L.D. Benson (western soapberry); P; T; UWL;

KES-246

\section{Sapotaceae}

Sideroxylon lanuginosum Michx. (chittamwood); P; T; UWL; KES-237

\section{Smilacaceae}

Smilax bona-nox L. (greenbrier); P; F; UWL; KES-264

Smilax rotundifolia L. (roundleaf greenbrier); P; F; UWL; KES-300

Smilax tamnoides L. (bristly greenbrier); P; F; UWL; KES-350

\section{Solanaceae}

*\#Lycium barbarum L. (common matrimonyvine); P; S; unknown habitat; KES-427

Physalis cinerascens (Dunal) Hitchc. (smallflower groundcherry); P; F; GL; KES-260

Solanum dimidiatum Raf. (horsenettle); P; F; GL; KES-262

Solanum elaeagnifolium Cav. (silverleaf nightshade); P; F; GL; KES-263

Solanum rostratum Dunal (buffalo bur); A; F; DA; KES-225

\section{Ulmaceae}

Ulmus americana L. (American elm); P; T; SBL; KES-238

*UImus pumila (Siberian elm); P; T; DA; KES-437

Ulmus rubra Muhl. (slippery elm); P; T; SBL; KES-387

\section{Urticaceae}

Parietaria pensylvanica Muhl. ex Willd. (Pennsylvania pellitory); A; F; SBL; KES-243

\section{Valerianaceae}

Valerianella amarella (Lindh. ex Engelm.) Krok (hairy cornsalad); A; F; GL; KES-257

Valerianella radiata (L.) Dufr. (beaked cornsalad); A; F; GL; KES-258

\section{Verbenaceae}

Glandularia pumila (Rydb.) Umber (pink mock vervain); A; F; GL; KES-256

Phyla lanceolata (Michx.) Greene (lanceleaf fogfruit); P; F; HWV; KES-254

Verbena bracteata Cav. ex Lag. \& Rodr. (bigbract verbena); A; F; GL; KES-232

Verbena halei Small (slender verbena); P; F; GL; KES-255

\section{Violaceae}

Viola bicolor Pursh (Johnny jump-up); A; F; GL; KES-253

Viola sororia Willd. var. missouriensis (Greene) L.E. McKinney (common blue violet); P; F; MF; KES-252 
Volume 16, December 2016

\section{Vitaceae}

Ampelopsis cordata Michx. (heartleaf peppervine); P; V; SBL; KES-388

Parthenocissus quinquefolia (L.) Planch. (Virginia creeper); P; V; SBL; KES-244

Vitis cinerea (Engelm.) Engelm. ex Millard (graybark grape); P; V; SBL; KES-251

Vitis vulpina L. (frost grape); P; V; SBL; KES-302

\section{Xanthorrhoeaceae}

*Hemerocallis fulva (L.) L. (orange daylily); P; F; SBL; KES-440 